\title{
Position and rotation of driver's head as risk factor for whiplash in rear impacts
}

\author{
James Lenard Karthikeyan Ekambaram Andrew Morris
}

7 April 2015

Loughborough
University

\begin{abstract}
Objective. There is evidence in the literature on whiplash that having the head turned at impact presents an increased risk of injury to vehicle occupants in rear impacts. The aims of this study were to collect exposure data on head position and rotation in a series of nine naturalistic driving trials and to express this in the form of a parametric statistical model that could be used in computer simulations to optimise seat design for the mitigation of soft-tissue neck injury.

Method. An instrumented vehicle equipped with an eye-tracker was used to collect digital readings that were complemented with a four-track video recording. The driving trials which each lasted 30-60 minutes were filtered for analysis to periods when the vehicle was stopped, stopping or moving slowly in accordance with accident data that indicated when rear impacts leading to neck injury were most likely to occur, amounting to around 75 minutes.

Results. It was found that the $t$ location-scale distribution provided the best fit to the experimental data. The measured interquartile range or central $50 \%$ of head movement that the drivers exhibited while most likely to incur neck injury was approximately $\pm 15 \mathrm{~mm}$ lateral, $\pm 10 \mathrm{~mm}$ longitudinal and \pm 7.5 degrees left-right rotation. These ranges also provide guidance to the degree of biofidelity required in computer models of the occupants. A manual review of video of the drivers' activities showed that out-of-range head rotation and rapid rotation explained the majority of missing digital readings from the eye-tracker whose operation was based on real-time image processing.

Conclusion. Exposure data on head position and rotation measured using an eye-tracker was summarised in parametric form and can be supplemented by models of extreme and rapid head rotation to account for missing data. Future research could aim for technical improvements in the range of measurement of the eye-tracker and proportion of missing readings and consolidate the results with a larger sample of drivers, vehicles and routes.
\end{abstract}




\section{Introduction}

Prevention of whiplash injuries remains an outstanding challenge in vehicle accident research. As yet, there is still uncertainty as to which lesion gives rise to whiplash symptoms so diagnosis and mitigation are subject to supposition. The same can be said for whiplash injury mechanisms although some evidence is available. Some studies suggest that having the head turned at impact is one of the risk factors for whiplash. For example, Barnsley and Bogduk (1994) reported a mechanism of injury in which impact to the rear of a vehicle causes an occupant's head (if it is already slightly rotated), to rotate further before neck extension occurs, pre-stressing various cervical spinal structures and increasing their susceptibility to injury. Sturzenegger et al. (1995) assessed 117 patients for long-term whiplash and also found head position to be statistically significant. They postulated as a mechanistic basis that the permitted range of extension of the neck is reduced by half when it is rotated. They also referred to further experiments with cadavers showing that the anterior longitudinal ligament was more liable to rupture when the head was rotated before application of an extension strain to the neck.

Some compilations of accident case files (Olsson et al., 1990; Jakobsson, 2004) have recorded whether occupants were actually turning their heads to the side at the time of impact. Jakobsson (2004) concluded that "Sitting posture, such as turned head and increased head to head restraint distance, significantly increases AIS 1 neck injury rates". Winkelstein et al. (2000) suggested that "axial pre-twist of the head and neck increase facet capsular strain and may play a role in the whiplash injury mechanism". Jakobsson et al. (2008) reported that "Occupants responding that they had turned their head (rotated around z axis to any degree) at the time of impact had a statistically significant higher risk of initial as well as persistent neck symptoms compared to those facing straight forward". Kumar et al. (2005) however struck a precautionary note based on tests of 20 healthy volunteers, not finding evidence that rotation of the head at impact necessarily increases the risk of neck injury, conjectured that "having the head rotated at the time of impact may be protective, just as expecting an impact may be protective against injury" although "with higher velocity impacts these protective factors may be overcome, resulting in more serious injury". The notion of reduced risk was not supported by Bunketorp and Elisson (2012) who observed that although the study of Kumar et al. did not find a greater injury risk when the head was rotated in their summary of electromyographic investigations, their data could only be used to evaluate the muscular reactions, not the load on the cervical spine.

Laboratory tests on specimens of the human spine have also provided mixed results. Maak et al. (2006) found that "the dynamic strains of the alar, transverse, and apical ligaments during impact did not exceed the corresponding non-injurious baseline values" while Siegmund et al. (2008) reported that an axial rotation "doubles the MPS in the capsular ligament compared to the neutral posture". They also found that capsular strains during the simulated whiplash exposure with the head turned were not significantly different from the 
maximum principal strain (MPS) associated with partial failure of the capsule.

Observational studies of head position and posture in vehicles have been undertaken by Parkin et al. (1995), Chapline et al. (2000) and Park et al. (2000). These three studies featured a relatively large number of subjects but only a snapshot of their seating position at one moment of time that was taken to represent the driver's normal condition. The relative position of the head and head restraint was measured along the longitudinal axis of the car but no account was made of head rotation in any of the studies. More recently, Jonsson et al. (2008) and Shugg et al. (2011) used observational recording instrumentation in the car to improve accuracy but also did not measure head rotation.

The design of seats, including the head restraint and associated safety technologies, should ideally be optimised across the range of postures that occupants exhibit at the moment of impact. This includes translational movements and rotations of the head. A natural approach to optimising seat design for a range of conditions is to run computer simulations of the interaction between the occupant and seat under crash conditions thereby obtaining a prediction of the dynamic load on the spine. At present the refinement of numerical models of the occupant to a high level of biofidelity in the spinal region poses a technical challenge; nevertheless with recent work in the area (e.g. Linder, 2011) and developments in computing power and digital resources, the capability to evaluate the performance of seats and anti-whiplash technologies using computer simulations will be achieved in the foreseeable future. At this time exposure data will be necessary to account for head rotation and occupant posture in the design of anti-whiplash systems.

In accordance with this requirement and to promote the ergonomic design of vehicle seats for occupant safety, the aims of this study were (a) to describe the position and rotation of drivers' heads in naturalistic driving under conditions when rear impacts tend to occur and (b) to summarise the experimental data in a parametric statistical model suitable for use in computer simulations.

\section{Methods}

\section{Participants}

Nine volunteers were available for the study (Table 1), each of whom drove for around 30-60 minutes through a designated route accompanied by an experimenter. In the first series of trials (subjects 1-4, route 1), travel directions were provided verbally by the experimenter while in the second series (subjects $5-9$, route 2) travel directions were provided by a portable navigation device

mounted on the dashboard. The volunteers were Loughborough University staff members and associates. 
Table 1: Age and sex of subjects in naturalistic driving trials.

\begin{tabular}{cclc} 
Subject & Age & Sex & Route \\
\hline 1 & 53 & male & 1 \\
2 & 35 & female & 1 \\
3 & 52 & male & 1 \\
4 & 41 & female & 1 \\
5 & 44 & male & 2 \\
6 & 49 & female & 2 \\
7 & 31 & male & 2 \\
8 & 24 & female & 2 \\
9 & 23 & male & 2 \\
\hline
\end{tabular}

Table 2: Manoeuvre of vehicles with rear impact resulting in neck strain to occupants $(\mathrm{N}=344)$.

\begin{tabular}{lr} 
Vehicle manoeuvre & $\%$ \\
\hline Waiting to go ahead but held up & 39 \\
Stopping on carriageway & 20 \\
Driving along straight road & 15 \\
Stopped waiting to turn & 9 \\
Driving in slow moving traffic & 4 \\
Other & 13 \\
\hline Total & 100 \\
\hline
\end{tabular}

\section{Apparatus}

The vehicle used for the trials, a 2010 Ford Mondeo sedan, was fitted with three main test instruments: a data logger for speed, acceleration and satellite location (GPS); an eye-tracker (faceLAB ${ }^{\mathrm{TM}} 5$ ) for head position and rotation; and a four-track video system.

\section{Driving route}

A review of in-depth accident data (Cuerden et al., 2008) indicated that occupants in passenger cars most often receive neck strain in rear impacts while stationary or moving relatively slowly in stop-go or congested traffic (Table 2). This guided the choice of a route for the trials passing through Leicester, England, a city with a population of over 300,000 . Following experience that the traffic density on the routes leading into and out of the city was too light to provide conditions highly relevant to the possible occurrence of a rear collision, the route was shortened to run entirely through the urban and suburban areas of the city. 


\section{Procedure}

The duration of the nine driving trials was over 60 minutes for trials 1-4 (route 1) and over 30 minutes for trials 5-9 (route 2). The periods when they were braking or stationary, and therefore considered to be at greater risk of rear impact, lasted around 8-18 minutes across the nine trial runs. The digital readings from the vehicle data logger and the eye-tracker were filtered to periods of interest by categorising each moment of driving as 'stopped', 'stopping' or 'other'. 'Stopped' was defined as a continuous period of at least one second when the vehicle speed was under $8 \mathrm{~km} / \mathrm{h}$ and 'stopping' was defined as a period of continuous deceleration leading to being 'stopped'. The $8 \mathrm{~km} / \mathrm{h}$ threshold was an arbitrary low speed, comparable to (brisk) walking pace and consistent with stop-go or slow moving traffic. Sections of the trials where the vehicle was 'stopped' or 'stopping' were then selected for detailed analysis because of their relevance to whiplash injury.

Operation of the eye-tracker was based on real-time image processing of facial features captured from two rearward-facing cameras mounted on the dashboard in front of the driver. Digital data could be lost when the head was rotated widely to the side or rotated rapidly from side to side disrupting the capability of the device to continuously track facial features. For this reason the analysis of the instrument readings was complemented by a review of the video recording to obtain a qualitative assessment of head movement. Focus was placed on periods of driving when the vehicle was stopped or stopping.

Data was extracted from the eye-tracker as text files and processed using PostgreSQL 9.2.3 and MATLAB R2013a. The data tables included a state variable that registered at each instant whether the image-processing algorithm had fixed on the facial features of the driver. Data readings during moments or periods of time when the algorithm was "searching" for the features were categorised as missing. In addition, preference was given in analysis to statistical parameters such as the median and quantiles that are insensitive to outlying values. The video analysis was conducted in slow-motion replay using a proprietary playback function supplied with the eye-tracker that linked the video frame number to the digital data (Seeing Machines, 2006). Glances to the internal and external mirrors, instrument panel, passenger and exterior were recognized by the direction of the head and detail of the eyes.

\section{Data analysis}

The eye-tracker recorded the position and rotation of the head on three axes (relative to the vehicle): longitudinal, lateral and vertical. Of the six resulting parameters, some were correlated in a predictable manner, for example rotation on the vertical axis (looking to the side) was associated with a lateral movement of the face while leaning sideways related a lateral movement of the head with a rotation on the longitudinal axis (a sideways tilt). These physically based correlations (which arise for example from the fixation of the base of the spine on the seat cushion) will automatically appear in any realistic computer simulation. 
Data analysis was therefore simplified by concentrating on three independent measures with a large range during driving: displacement in the longitudinal and lateral directions and rotation (left-right) on the vertical axis.

In order to use experimental readings for the optimisation of seat design using computer simulation, it is convenient to have the data modelled in parametric form. More than twenty forms of statistical distribution were considered, of which the t location-scale distribution stood out for the quality of fit to the experimental results. This distribution has the density function

$$
\frac{\Gamma\left(\frac{\nu+1}{2}\right)}{\sigma \sqrt{\nu \pi} \Gamma\left(\frac{\nu}{2}\right)}\left(\frac{\nu+\left(\frac{x-\mu}{\sigma}\right)^{2}}{\nu}\right)^{-\frac{\nu+1}{2}}
$$

with gamma function $\Gamma(\alpha)=\int_{0}^{\infty} e^{-t} t^{\alpha-1} \mathrm{~d} t$, location parameter $\mu$, scale parameter $\sigma$ and shape parameter $\nu$ (MathWorks, 2011). This distribution is considered useful for modelling data distributions that are more prone to outliers than the normal distribution; smaller values of $\nu$ yield heavier tails while at larger values of $\nu$ the $\mathrm{t}$ location-scale distribution approaches the normal distribution.

Further details of the vehicle instrumentation, driving routes, and six measured parameters are provided in Schick et al. (2011).

\section{Results}

The durations of the driving trials are shown in Figure 1. Missing readings (when the eye-tracker was not able to fix on facial features to quantify head position and rotation) are outlined at the top of each bar and shaded in yellow where the video was reviewed manually to categorise head movement (c.f. Figure 6 below). The proportion of missing readings varied widely from almost negligible in case 2 to over half in case 6 .

The median and interquartile range of lateral head position for the nine drivers while their vehicle was stopped or stopping is shown in Figure 2. There was a bias towards the left, i.e. centre of the passenger compartment. The interquartile range lies within $0-50 \mathrm{~mm}$ left of centre for seven of the nine cases and the median value was close to zero (centred) in the other two cases.

The median and interquartile range of longitudinal head positions for the nine drivers while their vehicle was stopped or stopping is shown in Figure 3. The interquartile range lies within around $25 \mathrm{~mm}$ for each subject while the median value varied considerably between drivers reflecting their preferred seating distance from the steering wheel and foot pedals, with subject 8 adopting the most forward position.

The position of the head restraint was identified using the eye-tracker by asking the subjects to rest their heads lightly against the head restraint for a few seconds before or after each trial run. The values recorded (Table 3) are in each case somewhat higher than the recorded range of movement, consistent with the head restraint presenting a physical obstruction to further backward 


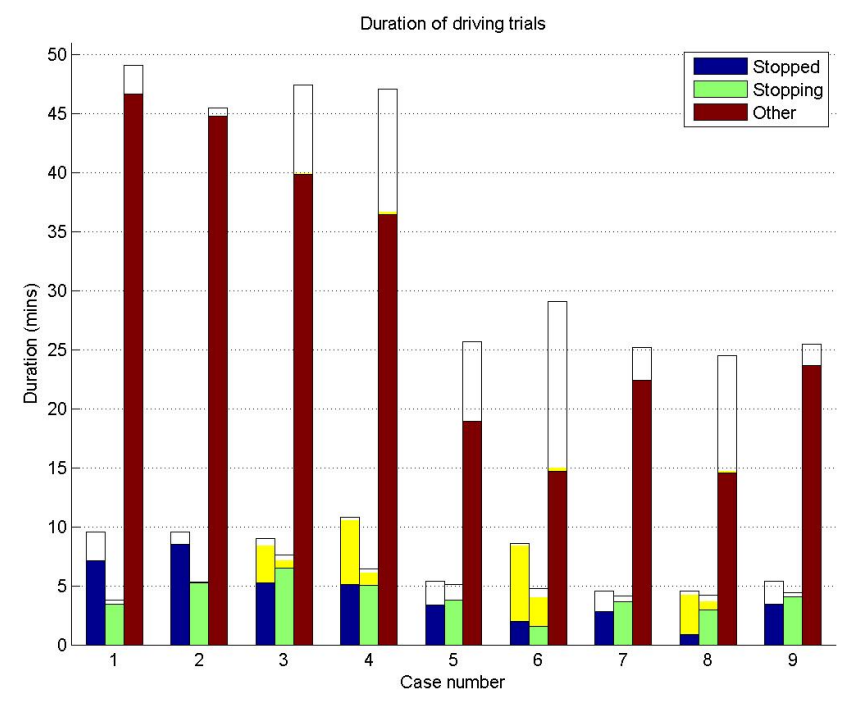

Figure 1: Duration of driving trials and vehicle movement for nine subjects. Missing instrument readings are shown in outline and shaded yellow where video reviewed manually.

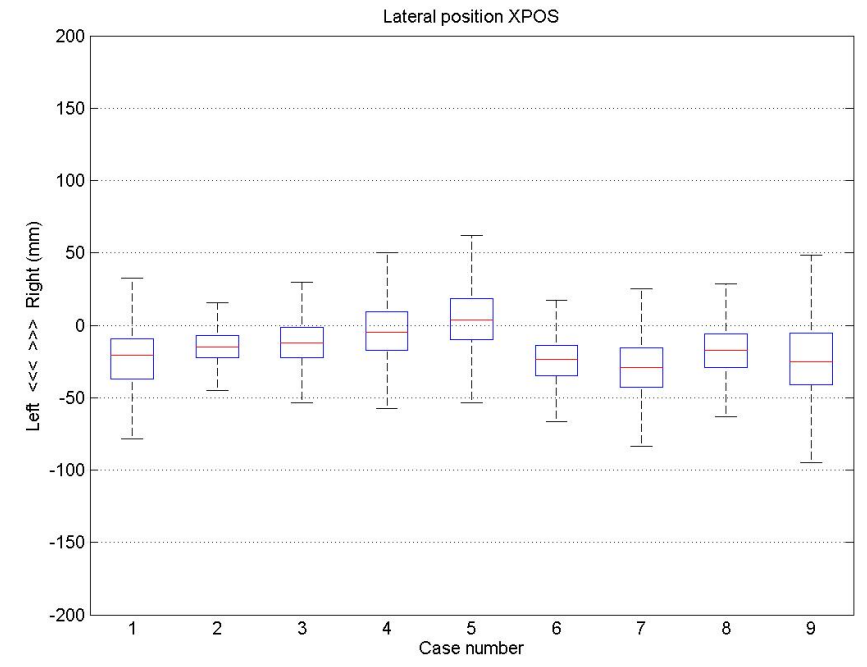

Figure 2: Lateral displacement of head for nine drivers while vehicle stopped or stopping. 


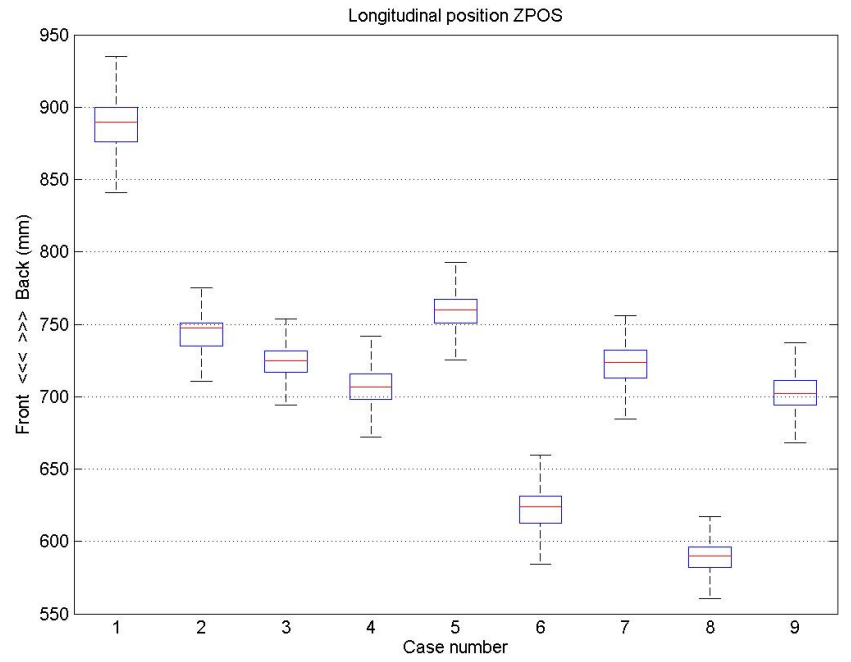

Figure 3: Longitudinal displacement of head for nine drivers while vehicle stopped or stopping.

Table 3: Reference position of head against head restraint for assessment of backset.

\begin{tabular}{cr} 
Subject & Position $(\mathrm{mm})$ \\
\hline 1 & 939 \\
2 & 790 \\
3 & 773 \\
4 & 776 \\
5 & 808 \\
6 & 668 \\
7 & 780 \\
8 & 640 \\
9 & 825 \\
\hline
\end{tabular}




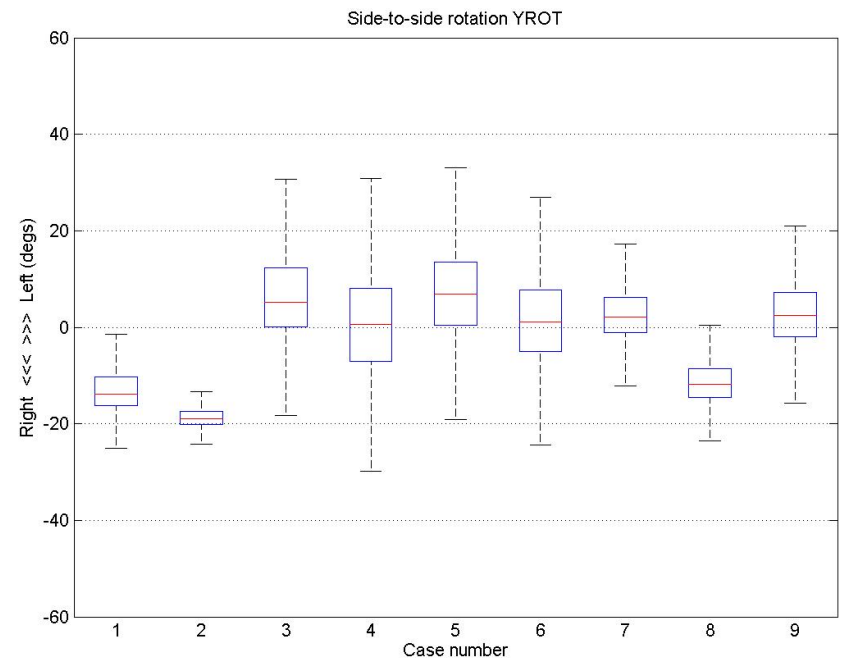

Figure 4: Rotation (left-right) of head for nine drivers while vehicle stopped or stopping.

movement. Thus backset (defined as the distance between the back of the head to the front of the head restraint) could be calculated. While not the focus of this study, backset is a parameter of traditional interest to seat designers in the context of soft-tissue neck injury. Taking case 1 as an example, the position of the head on the longitudinal axis had a median value of $886 \mathrm{~mm}$ while driving and a value of $939 \mathrm{~mm}$ while rested against the head restraint; the median value of dynamic backset was therefore $53 \mathrm{~mm}$.

The median and interquartile range of head rotation on the vertical axis (looking left-right) for the nine drivers while their vehicle was stopped or stopping is shown in Figure 4. The median value varied from close to twenty degrees towards the right (subject 2) to around eight degrees towards the left (subject 5 ) while the interquartile range varied from around three degrees (subject 2) to fifteen degrees (subject 4).

In order for the results presented in Figure 2, Figure 3 and Figure 4 to be used for the optimisation of seat design using computer simulation, it is most convenient to have the data modelled in parametric form. The t location-scale distribution stood out for its quality of fit to the experimental data. It is shown in comparison with a best-fit normal distribution for a single example that was fairly representative of the recorded data (Figure 5).

The experimental distributions typically had shorter tails than a Gaussian (normal) distribution. Longitudinal position was more skewed than the other parameters, consistent with the limitation of movement towards the back of the vehicle presented by the head restraint. The parametric values that provided a 


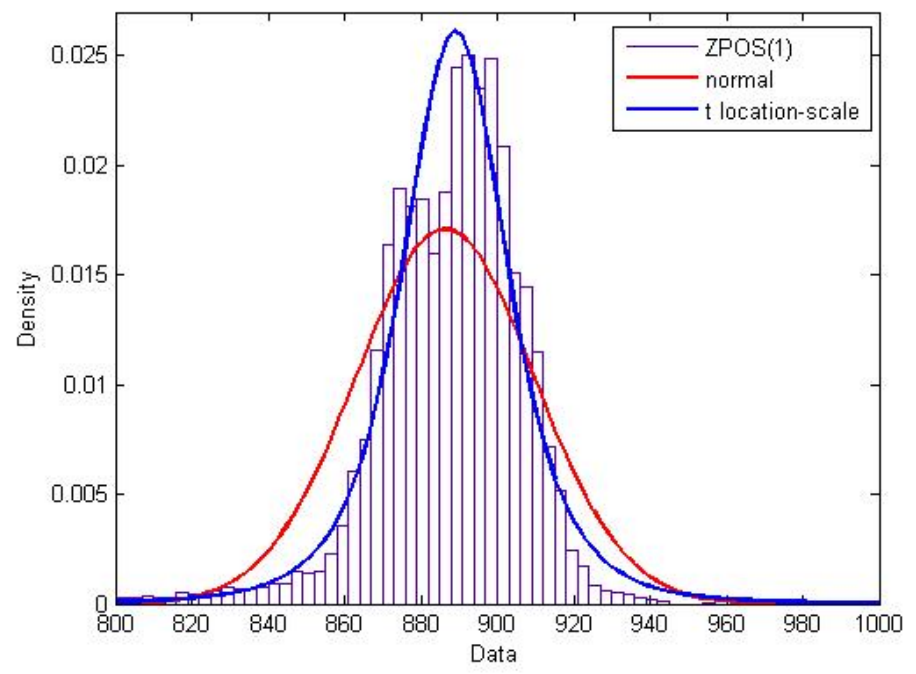

Figure 5: Example of modelling the instrument readings with normal (red) and $t$ location-scale (blue) distributions: position of head on longitudinal axis (subject 1).

Table 4: Parameters of $\mathrm{t}$ location-scale model for head position and rotation while vehicle stopped or stopping.

\begin{tabular}{c|rrr|rrr|rrr} 
Subject & $\mu$ & $\sigma$ & $\mathrm{N}$ & $\mu$ & $\sigma$ & $\mathrm{N}$ & $\mu$ & $\sigma$ & $\mathrm{N}$ \\
\hline 1 & -21.90 & 18.10 & 4.33 & 889.00 & 14.25 & 3.60 & -13.78 & 3.54 & 2.37 \\
2 & -14.83 & 9.46 & 1.90 & 748.25 & 5.17 & 1.16 & -18.94 & 1.42 & 1.09 \\
3 & -12.10 & 14.62 & 4.09 & 723.93 & 9.97 & 7.07 & 5.71 & 8.36 & 2.81 \\
4 & -4.71 & 19.26 & 87.41 & 706.83 & 12.20 & 5.40 & 0.33 & 10.92 & 6.02 \\
5 & 4.12 & 20.84 & 7.90 & 759.69 & 10.67 & 3.05 & 6.89 & 8.83 & 5.23 \\
6 & -24.24 & 14.47 & 8.34 & 623.02 & 12.30 & 3.93 & 1.21 & 8.41 & 2.60 \\
7 & -29.44 & 18.26 & 3.36 & 723.17 & 11.99 & 4.70 & 2.26 & 4.65 & 2.13 \\
8 & -17.38 & 16.38 & 14.39 & 589.09 & 9.66 & 5.05 & -11.73 & 3.72 & 2.32 \\
9 & -24.56 & 23.88 & 3.21 & 702.38 & 11.60 & 5.31 & 2.58 & 6.33 & 2.89 \\
\hline
\end{tabular}




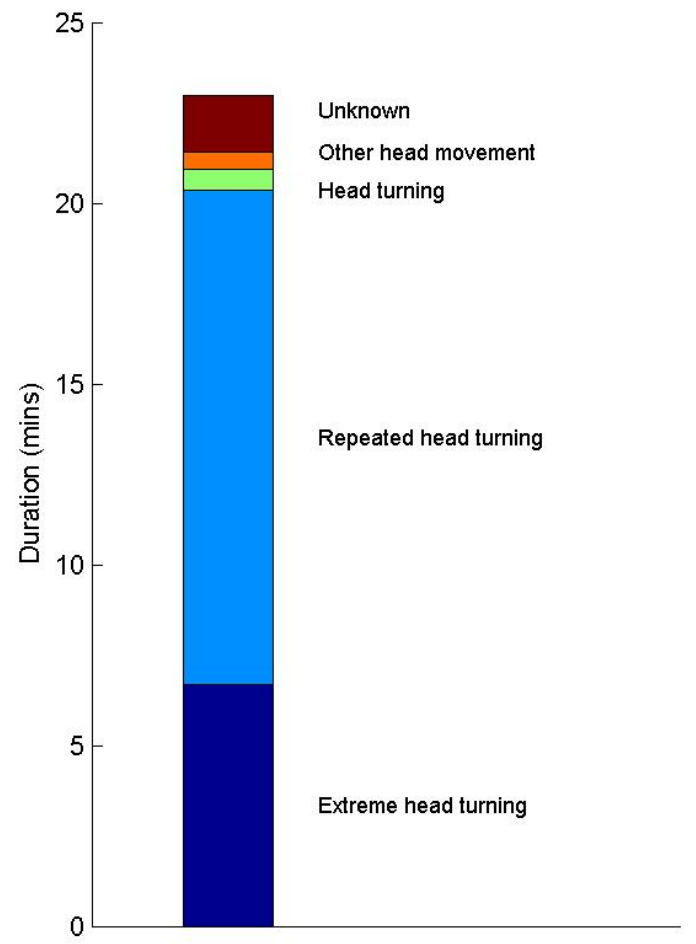

Figure 6: Activity of drivers obtained from video review while vehicle stopped or stopping and digital instrument readings not captured.

best fit to the experimental data are presented in Table 4 . These can be used directly or adapted for use in computer simulations.

Approximately 23 minutes of video were manually reviewed for the four drivers with the highest proportion of missing readings while their vehicle was stopped or stopping. This video review clarified the activity of drivers during the periods of missing data within the resources available for the work. Two types of activity were observed to provide the main explanation for the missing data: firstly rotation of the head beyond the measurable range of the eye-tracker and secondly rotation of the head rapidly from side to side, not necessarily beyond the range of measurement of the eye-tracker, but too fast for it to maintain continuous, real-time image processing. These are labelled as 'extreme head turning' ( 7 minutes) and 'repeated head turning' (13 minutes) in Figure 6. The explanation for missing readings in the remaining 2-3 minutes was either 'other types of head movement' or unknown. 


\section{Discussion}

The median values of lateral head position tended to be left of centre and the median values of head rotation were also off-centre (non-zero) in several cases. This phenomenon is thought to be real. Subjects were observed to adopt asymmetrical driving postures and sometimes appeared to focus their attention towards objects and activities on the side of the road, especially in urban areas. Furthermore the driver's side window and door obviously restrict lateral movement in that direction while some of the controls that the driver may reach for while the vehicle is stationary are located on the centre console. These asymmetries may have implications for the optimal position and width of the head restraint.

The dynamic measurement of longitudinal head position and, by implication, backset raises the question of how best to define this important parameter. The median value while driving - the natural choice in this context-may not be identical to the static position taken up on request by subjects in other studies.

The eye-tracker generated a proportion of missing readings across all phases of the naturalistic driving trials for reasons that were fairly identifiable, including ambient light fluctuations and physical obstruction of the line of sight between camera and face. Of greater significance to this study were missing readings due to specific types of head movements because of the bias this could introduce to the results. The video review indicated that in fact two types of conditions are probably under-represented in the digital readings; (a) having the head turned to the side at an angle that is out of the range of measurement of the face-tracker as configured in the trials and (b) a relatively flat distribution of angles from wide left to wide right that is lost because of a rapid speed of motion which is a characteristic of looking in both directions for an opportunity to pull out into a carriageway. It is suggested that these two conditions could be modelled independently in computer simulations as an adjunct to the parameters of the t location-scale model.

A primary aim of this study was to clarify the range of conditions under which seat performance should be optimised to mitigate the risk of neck injury. A further outcome to the study, given that computer models of occupants are under continuing development, is that the results also indicate the range of biofidelity that is desirable in the computer models. To accommodate for example $50 \%$ of the range of head movement that drivers exhibit while most likely to incur neck strain in a rear impact, it would on the basis of the information available be necessary to deal with approximately $\pm 15 \mathrm{~mm}$ lateral movement, \pm 10 $\mathrm{mm}$ longitudinal movement and \pm 7.5 degrees left-right rotation of the head.

The degree of variation between subjects particularly exemplified in Figure 2, Figure 3 and Figure 4 and summarised in Table 4 was not known until the experimental program had been carried out. It was considered statistically inadvisable to combine the cases into a group analysis on the present number of cases given the level of inter-subject variability and the unequal duration of 'stopped or stopping' periods in the naturalistic driving trials. It is suggested

that when the statistical parameters of the t location-scale model are used in computer simulation to appropriately weight the position of the driver's head at 
impact, a choice should be made at that stage whether to optimise the seat for one or two representative drivers or across the full range of variation indicated by this study.

The core results of the seat posture driving trials ultimately derive from nine subjects driving a single vehicle on two routes through a single city for 75 minutes. It would be unsafe to recklessly extrapolate the findings to a wider population of drivers, vehicles, routes or cities. The value of this study lies in sketching the outlines of a picture about which little or no information was previously available: quantifying the range of head movement as a risk factor for whiplash-associated disorders among car drivers under traffic conditions when a rear impact could occur. The degree of similarity among the subjects lends a qualified confidence to the expectation that the results would be consistent with the outcome of a wider, deeper or more diverse study.

\section{Conclusions}

Of the three parameters described in detail, lateral head position demonstrated most uniformity of median value and interquartile range for the nine subjects; longitudinal position showed uniformity of the interquartile range but wide differences in the median value; while left-right rotation showed considerable differences in both the median value and interquartile range. Incorporating these three main independent movements into a computer model of a seated human body or crash test dummy using the statistical parameters provided would produce an effective first-order simulation of a driver's posture while in control of a vehicle. This would include posture under traffic conditions associated with a risk of soft-tissue neck injury from rear impact. Data would enable the design of seats to be optimised for the mitigation of whiplash taking into account head position and rotation as an aggravating risk factor.

Technical improvements for future studies of this type should aim at a reduction in the proportion of missing readings from the eye-tracker and an increase in its range of measurement, particularly side-to-side rotation. A larger sample of drivers, vehicles and routes would be beneficial in future research to consolidate the results of this pioneering study and to obtain a more detailed correlation of head position with specific vehicle manoeuvres and traffic situations.

\section{References}

L. Barnsley and N. Bogduk. Whiplash injury. Pain, 58(3):283-307, 1994.

O. B. Bunketorp and L. K. Elisson. Cervical status after neck sprains in frontal and rear-end car impacts. Injury, 43(4):423-430, 2012.

J. F. Chapline, S. A. Ferguson, R. P. Lillis, A. K. Lund, and A. F. Williams. Neck pain and head restraint position relative to the driver's head in rear-end collisions. Accident Analysis and Prevention, 32(2):287-297, 2000. 
R. Cuerden, M. Pittman, E. Dodson, and J. Hill. The UK On-the-Spot accident data collection study - phase II report. Report for the Department for Transport Road Safety Research Report no. 73, 2008.

L. Jakobsson. Field analysis of AIS1 neck injuries in rear-end car impactsinjury reducing effect of the WHIPS seat. Journal of Whiplash and Related Disorders, 3(2):37-53, 2004.

L. Jakobsson, I. Isaksson-Hellman, and M. Lindman. WHIPS (Volvo Cars' whiplash protection system) - the development and real-world performance. Traffic Injury Prevention, 9:600-605, 2008.

B. Jonsson, H. Stenlund, and U. Björnstig. Backset - stationary and during car driving. Traffic Injury Prevention, 9(6):568-573, 2008.

S. Kumar, R. Ferrari, and Y. Narayan. Effect of head rotation in whiplash-type rear impacts. Spine, 5(2):130-139, 2005.

S. Linder. Tool for evaluation of adaptive seat to reduce neck injuries for female and male occupants. In IQPC 6th International Conference Innovative Seating, Frankfurt, Germany, 2011.

T. G. Maak, Y. Tominaga, M. M. Panjabi, and P. C. Ivancic. Alar, transverse, and apical ligament strain due to head-turned rear impact. Spine, 31(6): 632-638, 2006.

MathWorks. Statistics toolbox for use with MATLAB ${ }^{\text {TM}}$ : user's guide. Technical report, The MathWorks Inc., Massachusetts, 2011.

I. Olsson, O. Bunketorp, G. Carlsson, C. Gustafsson, I. Planath, H. Norin, and L. Ysander. An in-depth study of neck injuries in rear end collisions. In Proceedings 1990 International IRCOBI Conference on the Biomechanics of Impacts, Lyon, France, 1990.

S. J. Park, C. B. Kim, C. J. Kim, and J. W. Lee. Comfortable driving postures for Koreans. International Journal of Industrial Ergonomics, 26(4):489-497, 2000 .

S. Parkin, G. M. Mackay, and A. Cooper. How drivers sit in cars. Accident Analysis and Prevention, 27(6):777-783, 1995.

Sylvia Schick, Wolfram Hell, Nathalie Gales, Anders Kullgren, Helena Stigson, Lotta Jakobsson, Kai-Uwe Schmitt, Ernst Tomasch, and James Lenard. ADSEAT deliverable 1.1: Real world data. Report for European Commission, November 2011.

Seeing Machines. FaceLAB 4 user manual. Technical report, Canberra, 2006.

J. A. Shugg, K. Vernest, and J. P. Dickey. Head restraint backset during routine automobile driving: drivers usually exceed the recommended guidelines. Traffic Injury Prevention, 12(2):180-186, 2011. 
G. P. Siegmund, M. B. Davis, K. P. Quinn, E. Hines, B. S. Myers, S. Ejima, K. Ono, T. Kamiji, T. Yasuki, and B. A. Winkelstein. Head-turned postures increase the risk of cervical facet capsule injury during whiplash. Spine, 33 (15):1643-1649, 2008.

M. Sturzenegger, B. P. Radanov, and G. di Stefano. The effect of accident mechanisms and initial findings on the long-term course of whiplash injury. Journal of Neurology, 242(7):443-449, 1995.

B. A. Winkelstein, R. W. Nightingale, W. J. Richardson, and B. S. Myers. The cervical facet capsule and its role in whiplash injury: a biomechanical investigation. Spine, 25(10):1238-1246, 2000. 